\title{
Proceedings of the First South American Oral Health Panel
}

\section{Cláudio Mendes Pannuti(a) \\ (a) Biodentistry Graduate Program, School of Dentistry, Ibirapuera University, São Paulo, SP, Brazil.}

$\mathbf{T}_{1}^{\mathrm{h}}$ he papers in this supplement were presented at a Symposium at the Latin American Federation for Dental Research Meeting, held in Mar del Plata in November 2005. The Symposium, entitled "A Major Overview of the Oral Healthcare Status in South America", had the objective to present the consensus of a group of prominent Latin American researchers and their recommendations for oral health in South America. The symposium and this supplement have been sponsored by Pfizer Consumer Healthcare.

The Symposium attendees were:

- Professor Silvia Lopez de Blanc (Chair), National University of Cordoba, Argentina.

- Professor Rui Vicente Oppermann (Co-Chair), Federal University of Rio Grande do Sul, Brazil.

- Professor Andrew Tawse-Smith, Colegio Odontologico Colombiano, Colombia.

- Professor José Roberto Cortelli, University of Taubaté, Brazil.

- Professor Raul Emilio de La Sotta Thénoux, Universidad Central de Chile, Chile.

- Professor Victoria Eugenia Criado Montoya, Universidad Central de Venezuela, Venezuela.

In the first paper, Dr. Oppermann provided an overview of the epidemiology of periodontal diseases in Latin America, and discussed the influence of risk factors on this population. Due to the scarcity of data from the region, the author concludes that epidemiological research should be a priority in Latin America.

A possible role of alcohol-containing mouthrinses in the etiology of oral cancer has been the subject of speculation for decades. The current knowledge about this association is summarized by Drs. Lopez and Baruzzi. Up to now there is no evidence of a causal relationship between the use of alcohol-containing mouthrinses and the development of oral cancer.

Drs. Cortelli and De La Sotta have reviewed the effect of antimicrobial mouthrinses containing active ingredients, such as chlorhexidine and essential oils against oral microorganisms.

Recent studies in South American countries have demonstrated a clear tendency in increasing rates of periodontal disease as the population ages. Dr. Tawse-Smith has explored the benefits of adjunctive antimicrobial mouthrinses on the prevention of root caries and periodontal diseases in elderly individuals, due to their limited capacity of removing dental plaque by mechanical methods. 
Finally, Drs. Criado and Tawse-Smith have explored the factors associated with lack of compliance with oral preventive programs. The limited compliance of the individuals suggests the importance of adjunctive aids, such as antiseptic mouthrinses, to enhance mechanical plaque control measures.

The main conclusions of the group were:

- Mechanical means of plaque control may not be sufficient to maintain oral health.

- Antiseptic mouthrinses should be used in addition to tooth brushing and flossing.

- Mouthrinses containing high concentration of chlorhexidine and essential oils present the high- er evidence of efficacy in the reduction of dental plaque and gingivitis.

- Chlorhexidine-containing mouthrinses have been related to extrinsic staining of the teeth and tongue, increased calculus formation and alteration of the taste sensation. Therefore, long-term use should be indicated for severe cases of periodontal disease and supervised by the dental professional, or recommended for short-term use only.

- Essential oils mouthrinses long-term use is safe and has not been associated with adverse effects. Therefore, the long-term use for the control of dental plaque and gingivitis is indicated. 